\title{
On the time required to construct a simple linear order
}

\author{
KIRK H. SMITH and BARBEE T. MYNATT \\ Bowling Green State University, Bowling Green, Ohio 49403
}

\begin{abstract}
Pairs of digits or two-syllable words were presented visually for subjects to study as long as necessary in order to construct a single string reflecting the ordering inherent in the pairs. For example, given $75,27,58$, the subject was to produce 2758 . The time spent studying individual pairs was shown to reflect a set of five constructive processes identified in a theory proposed by Foos, Smith, Sabol, and Mynatt (1976) to account for errors in constructing the correct order. In addition, the results indicate that the time required to determine that a pair contains no elements in common with previously presented pairs is greater for two-syllable words than for digits, suggesting that an exhaustive search through short-term storage is occurring.
\end{abstract}

One of the simplest versions of the linear order task consists of presenting a subject with a set of digit pairs and requesting that he respond with a single string of digits reflecting the order inherent in the presented pairs. For example, the subject might receive, one at a time, the pairs $72,28,83$, and his task would be to write down 7283 . A recent study by Foos, Smith, Sabol, and Mynatt (1976) found that the order of presentation of the pairs was a major determinant of success in this task. Subjects were successful $91 \%$ of the time with the presentation order given above, but succeeded only $46 \%$ of the time with an order such as $62,34,46$.

Foos et al. (1976) explained their results in terms of what they called constructive processes, consisting of sets of mental operations that subjects are assumed to perform on the input strings. A detailed discussion of the various mental operations may also be found in the section by Smith in Potts, Banks, Kosslyn, Moyer, Riley, and Smith (in press). Briefly, the theory asserts that the first pair is stored in a short-term memory buffer and that subsequent pairs are entered following a marker element at the end of the string in the buffer. So, for example, after hearing 91,15 , the buffer contains 91-15, where the hyphen ("-") represents the marker. The buffer is continually searched for duplicate, or matching, elements and new strings are constructed in the buffer by bringing duplicate elements together around a marker and deleting both the marker and one of the duplicate elements. Thus, 91-15 would become 915 in the buffer.

Five constructive processes varying in difficulty were identified. In increasing order of difficulty, they are, as

This research was supported by National Science Foundation Grant BMS75-19313. The second author is also on the faculty of the University of Toledo. Requests for reprints should be sent to Kirk H. Smith, Department of Psychology, Bowling Green State University, Bowling Green, Ohio 43403. follows: (1) Process M1: One matching element is found already arrayed on either side of the marker. Simple deletion operations produce a constructed order. (This process was illustrated above with the pairs 91,15 .) (2) Process M2: One matching element is found, but a rearrangement is required to bring the matching digits into proper order. For example, 64,26 is first represented in the buffer as $64-26$, then rearranged to form 26-64, and finally by deletion reduced to 264 . (3) Process $\mathrm{N}$ : No matching elements can be located. A complete search of the buffer is required, and no reduction in the contents of memory is possible. (4) Process D1 : Two different elements are duplicated, and the arrangement of elements in the buffer corresponds to the order that must be constructed. The sequence $57,91,79$ reflects both Process $\mathrm{N}$ after the second pair, with 57-91 remaining in the buffer, and Process D1 after the third pair. Note that the order to be constructed, 5791, is present in the buffer following the second pair. (5) Process D2: Again two matching elements are present, but the elements require rearrangement before the correct order can be achieved. The sequence 81, 2338 leads to Process D2 on the third pair. The contents of the buffer following the second pair will be $81-23$, and this must be rearranged to yield the correct order 2381 .

The foregoing account is very brief, but it should be clear that an order of presentation will be more difficult if it requires Process M2 or D2 than if it requires Process M1 or D1, respectively. The theory explains this difference in terms of an extra step in which the contents of the memory buffer are rearranged. Also, any presentation order that requires Process $\mathbf{N}$ will involve the temporary storage of extra elements as compared to orders that require only Processes M1 and M2. However, differences in error rate do not precisely indicate whether the added difficulty in the latter case is due to the increased searching and extra memory demands of Process $\mathrm{N}$ or to the rearrangement and deletion operations common to Processes D1 and D2. 
Foos et al. (1976) sought to make the error analysis more precise by assigning errors to the constructive process that was required when that pair was introduced. As shown in Table 1, when the presentation series $34,12,23$ occurs, Process D2 occurs on the third pair, 23. If the string was erroneously constructed as 3412,23 is taken to be in error and the error counted against Process D2. But it is obvious that such comparisons are founded on the assumption that difficulty with a given process affects retention and integration of the pair introduced. Clearly, the failure of a constructive process could result in the loss of previously stored relations or could even undo a previously constructed order.

In the present study, subjects saw the pairs on a visual display and were permitted to control how long they viewed each one. Given sufficient time to complete the necessary constructive processes, subjects were expected to arrive at the correct string on nearly every trial. However, the different mental operations involved in constructing the order were expected to produce differences in study time. These differences could then be used to establish the exact locus of difficulty in construction. For example, if, as Foos et al. have suggested, Process M2 is more difficult than Process M1 because the former requires a reordering operation not required in the latter, the study times on pairs involving Process $\mathrm{M} 2$ should be longer. Of special interest is the possibility that study-time data will reveal whether the difficulty of presentation orders involving Process $\mathrm{N}$ (nonmatch orders) is due to the failure to find a match (Process N) or to the subsequent scanning operations and symbol manipulations that are required (Processes D1 and D2).

There have also been several investigations of presentation order effects in linear-order problems using sentences to present the relationships between adjacent elements (Mynatt \& Smith, in press; Smith \& Foos, 1975). These studies used a small set of familiar profession names as elements. In order to determine whether such words were treated differently than digit names, we gave each of our subjects one set of trials on which all pairs were composed of digits and another set of trials on which word pairs were used.

\section{METHOD}

The pairs were presented on an Owens-Illinois Digivue plasma display screen under the control of a NOVA 1220 computer. The subject controlled the rate of presentation by pressing the space bar on the Digivue console to see each pair. Each trial consisted of either three or four pairs. On a counterbalanced half of the trials, the third presented pair was followed by a fourth that always introduced a new element to be added to the end of the four-term order implied by the first three pairs. The purpose of this manipulation was to prevent the subject from viewing the third pair just long enough to read it and then pressing for the recall signal without engaging in any constructive processes. Under the present procedure, the subjects never knew whether the third pair would be followed by a recall
Table 1

Mean Proportion of Errors as a Function of Presentation Order

\begin{tabular}{|c|c|c|c|c|}
\hline \multirow[b]{2}{*}{ Presentation Order } & \multirow[b]{2}{*}{ Input Pairs* } & \multicolumn{2}{|c|}{$\begin{array}{c}\text { Constructive Process } \\
\text { Involved** }\end{array}$} & \multirow[b]{2}{*}{ Errors } \\
\hline & & $\begin{array}{c}\text { After } \\
\text { 2nd } \\
\text { Pair }\end{array}$ & $\begin{array}{c}\text { After } \\
\text { 3rd } \\
\text { Pair } \\
\end{array}$ & \\
\hline \multicolumn{5}{|l|}{ Match Orders } \\
\hline 1 Forward & $\mathrm{AB}, \mathrm{BC}, \mathrm{CD}$ & M1 & M1 & .11 \\
\hline 2 Reverse & $\mathrm{CD}, \mathrm{BC}, \mathrm{AB}$ & M2 & M2 & .09 \\
\hline 3 Type 3 & $\mathrm{BC}, \mathrm{CD}, \mathrm{AB}$ & M1 & M2 & .08 \\
\hline 4 Type 4 & $\mathrm{BC}, \mathrm{AB}, \mathrm{CD}$ & M2 & M1 & .08 \\
\hline \multicolumn{5}{|l|}{ Nonmatch Orders } \\
\hline 5 Confirmation & $\mathrm{AB}, \mathrm{CD}, \mathrm{BC}$ & $\mathbf{N}$ & D1 & .06 \\
\hline 6 Disconfirmation & $\mathrm{CD}, \mathrm{AB}, \mathrm{BC}$ & $\mathbf{N}$ & D2 & .61 \\
\hline
\end{tabular}

*For purposes of illustration, the order to be constructed in every case is $A B C D$.

${ }^{* *}$ For details, see text and Foos et al. (1976).

signal or another pair. Study times for the fourth pair were not included in any analysis. After the third or fourth pair, the word "recall" appeared on the screen, at which time the subject was instructed to write down the array on a response sheet that provided horizontal lines for each answer. The time each pair was displayed was measured in milliseconds by the computer and recorded as the study time for that pair.

A trial block consisted of four presentations of each of the six possible four-term orders. These orders are shown in Table 1, where they are identified by the numbers and names used by Foos et al. (1976) and Smith and Foos (1975). In one block of 24 trials, array elements were the five two-syllable common words, doctor, farmer, leader, soldier, teacher (AA words, Thorndike-Lorge, 1944).

A second block of trials used the digits 1 through 9 as array elements. On each trial, either four or five different elements were randomly selected and ordered to make up the array. Each subject received a unique random order of the 24 trials in each block and a different randomly selected ordering of elements for each trial.

The subjects were eight undergraduate students who were required to participate in psychological research as part of a course in introductory psychology. Half of the subjects received the trial block with word elements before the trial block with digit elements, and the remaining subjects received the reverse order.

\section{RESULTS AND DISCUSSION}

The mean error rates for each presentation order are shown in Table 1 . The overall mean error rate (.17) was somewhat lower than that obtained by Foos et al. (.28). However, permitting subjects to pace their own presentation sequence clearly did not completely eliminate errors. Therefore, errors were analyzed in a fivefactor analysis of variance involving presentation order, type of element (digit or word), size of array (four or five digits), replications (two occurrences of the same presentation order), and groups. The latter differed in whether digits or words were presented first. The first four factors were repeated measures. (All tests were conducted at the .05 level of significance unless otherwise stated.) The error rate on trials involving five elements (.21) was significantly higher than that on trials 
involving four elements $(.14)[\mathrm{F}(1,6)=6.84, \mathrm{MSe}=$ $.075]$.

Presentation orders were also a significant source of variance $[F(5,50)=13.67, \mathrm{MSe}=2.16]$. Inspection of the mean proportions in Table 1 suggested that the error rate for Order 6 (disconfirmation) was greater than that of the other five orders, which did not differ. This impression was confirmed by Tukey's test (hsd $=.25$ ). There were two three-way interactions of Orders by Replications, one with type of element and the other with size of array. Both are essentially uninterpretable, although in neither case were the two main effects discussed above qualitatively different at various levels of the interacting factors.

The mean study times for each presentation order are shown as lines in Figure 1. The two types of elements, digits and words, have been plotted separately. Each point in the figure represents an individual input event, and the abscissa represents the first, second, and third pair presented.

Study times on the first pair were significantly shorter than on the second and third $[\mathrm{F}(1,7)=15.69$, $\mathrm{MSe}=53.41]$. This result is consistent with the theory in that no constructive processes occur when the first pair is presented. There were also no differences among study times on the first pair for different presentation orders $(\mathrm{F}<1)$.

The input events were grouped according to the constructive process (shown in Table 1) hypothesized to be occurring when each pair was presented. Mean study times for each of the five constructive processes are presented in Table 2 . These groups of input events were compared using appropriately weighted contrasts derived from an analysis of variance that treated the six presentation orders and the three pairs per trial as a single 18-level factor. Other factors in the analysis were type of element, digit or word, and replications; all were repeated-measures factors.

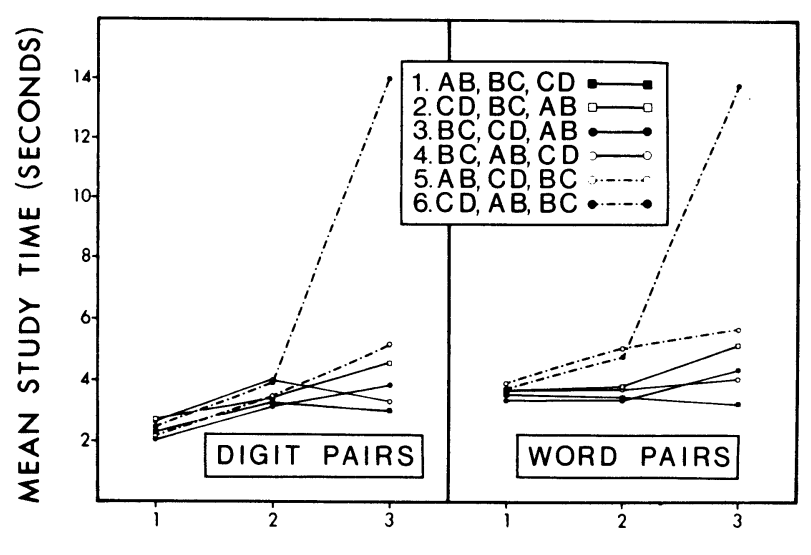

PAIR

Figure 1. Mean study time in seconds for individual pairs as a function of the ordinal position of a pair within a trial for the six presentation orders of four-term linear orders.
Table 2

Mean Study Time in Seconds as a Function of Constructive Process and Ordinal Position of the Pair

\begin{tabular}{ccc}
\hline Process & Second Pair & Third Pair \\
\hline M1 & 3.30 & 3.39 \\
M2 & 3.69 & 4.47 \\
N & 4.30 & \\
D1 & & 5.39 \\
D2 & & 13.88 \\
\hline
\end{tabular}

On the second and third pairs of match orders, study times were compared for input events involving Processes M1 and M2. Mean study time was significantly longer for M2 than for M1 $[\mathrm{F}(1,7)=7.64, \mathrm{MSe}=9.17]$. This result suggests that adding a new element to the front of a previously stored array of ordered elements requires $.74 \mathrm{sec}$ longer on the average than adding a new element to the end of such an array. The difference is consistent with the constructive theory, especially when it is noted that mean study times for match processes fall just beyond the 3-sec presentation rate used by Foos et al. Some of the errors in the latter study can be attributed to the fact that subjects did not always have sufficient time to complete the construction of a partial linear order before the arrival of another pair.

Mean study times for the third pair of nonmatch orders were also compared. Where Process D2 was involved, the study times were significantly greater than where Process D1 was involved $[F(1,7)=8.33$, $\mathrm{MSe}=276.77]$. This difference of $8.49 \mathrm{sec}$ is parallel to the large difference in error rates found by Foos et al. and suggests that the rearrangement operations postulated by them for Process D2 are very difficult to carry out without error, even when the subject has virtually unlimited time. It should also be noted that this difference was highly variable across subjects (ranging from $21 \mathrm{sec}$ to $-2 \mathrm{sec}$; only one subject had a negative difference). Although a planned comparison between $\mathbf{M}$ processes and $D$ processes was significant $[F(1,7)=$ $16.64, \mathrm{MSe}=167.03 \mathrm{]}$, it is clear that the difference is due primarily to the D2 process. A post hoc comparison of Process D1 with the match processes which occurred on the third pair did not approach significance.

Study times on the second pair were grouped into two sets, those for match and nonmatch processes. The contrast was marginally significant $(\mathrm{p}<.10)[\mathrm{F}(1,7)=$ $4.88, \mathrm{MSe}=11.22$ ]. However, this was the only comparison that showed a significant interaction with type of element $[\mathrm{F}(1,7)=8.03, \mathrm{MSe}=3.26]$. As can be seen in Figure 1, the nonmatch process required longer than the match process for word pairs, whereas this difference was very small for digit pairs. Overall study times for digits and words did not differ significantly $[F(1,7)=$ 1.67]. A pattern of results such as this might be expected if a scanning operation played an especially important role in Process $\mathrm{N}$ and the scanning rate was influenced by the number of syllables in the elements. Digits have, on the average, fewer syllables (only seven 
has two) than the five two-syllable profession names that were used. Since there are no duplicate elements to be found and manipulated in Process $\mathrm{N}$, it was regarded by Foos et al. (1976) as an exhaustive search and comparison process. Although search and comparison play a role in $\mathbf{M}$ and $\mathrm{D}$ processes, they were assumed to terminate after relatively few elements had been examined. Hence, the resulting interaction is consistent with the theory and supports the general claim that there are five distinct processes involved in the construction of linear orders.

\section{REFERENCES}

Foos, P. W., Smith, K. H., SAbol, M. A., \& Mynatt, B. T. Constructive processes in simple linear order problems.
Journal of Experimental Psychology: Human Learning and Memory, 1976, 2, 759-766.

Mynatt, B. T., \& Smith, K. H. Constructive processes in linear order problems revealed by sentence study times. Journal of Experimental Psychology: Human Learning and Memory, in press.

Potrs, G. R., Banks, W. P., Kosslyn, S. M., Moyer, R. S., Riley, C. A., \& SMIth, K. H. Encoding and retrieving ordered relationships. In N. J. Castellan \& F. Restle (Eds.), Cognitive theory (Vol. 3). Hillsdale, N.J: Lawrence Erlbaum, in press.

SmITH, K. H., \& Foos, P. W. Effect of presentation order on the construction of linear orders. Memory \& Cognition, 1975, 3, 614-618.

THORNDIKE, E. L., \& LORGE, I. The teacher's word book of 30,000 words. New York: Columbia University Press, 1944.

(Received for publication January 29, 1977.) 